International Journal of Pure and Applied Mathematics

Volume 83 No. 1 2013, 1-5

ISSN: $1311-8080$ (printed version); ISSN: 1314-3395 (on-line version)

url: http://www.ijpam.eu

doi: http://dx.doi.org/10.12732/ijpam.v83i1.1

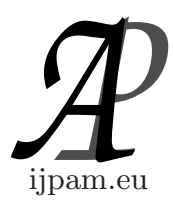

\title{
TUPLES OF OPERATORS AND SUPERCYCLICITY
}

\author{
B. Yousefi ${ }^{1}$, Gh.R. Moghimi \\ ${ }^{1,2}$ Department of Mathematics \\ Payame Noor University \\ P.O. Box 19395-3697, Tehran, IRAN
}

Abstract: In this paper, we give sufficient conditions for a tuple of operators to be supercyclic.

AMS Subject Classification: 47B37, 47B33

Key Words: tuple of operators, supercyclic vector

\section{Introduction}

By an n-tuple of operators we mean a finite sequence of length $n$ of commuting continuous linear operators on a Banach space $X$.

Definition 1.1. Let $\mathcal{T}=\left(T_{1}, T_{2}, \ldots, T_{n}\right)$ be an n-tuple of operators acting on an infinite dimensional Banach space $X$. We will let

$$
\mathcal{F}_{\mathbf{T}}=\left\{T_{1}{ }^{k_{1}} T_{2}{ }^{k_{2}}, \ldots, T_{n}{ }^{k_{n}}: k_{i} \geq 0, i=1, \ldots, n\right\}
$$

be the semigroup generated by $\mathcal{T}$. For $x \in X$, the orbit of $x$ under the tuple $\mathcal{T}$ is the set $\operatorname{Orb}(\mathcal{T}, x)=\left\{S x: S \in \mathcal{F}_{\mathrm{T}}\right\}$. A vector $x$ is called a hypercyclic vector for $\mathcal{T}$ if $\operatorname{Orb}(\mathcal{T}, x)$ is dense in $X$ and in this case the tuple $\mathcal{T}$ is called hypercyclic. Also, a vector $x$ is called a supercyclic vector for $\mathcal{T}$ if $\operatorname{COrb}(\mathcal{T}, x)$

Received: December 12, 2011

(C) 2013 Academic Publications, Ltd. url: www.acadpubl.eu

$\S_{\text {Correspondence author }}$ 
is dense in $X$ and in this case the tuple $\mathcal{T}$ is called supercyclic. The set of all supercyclic vectors for a tuple $\mathcal{T}$ is denoted by $S C(\mathcal{T})$.

Supercyclic operators were introduced by Hilden and wallen. They proved that all unilateral backward weighted shifts on a Hilbert space have a supercyclic vector. The study of supercyclic operators has experimented a great development during the last years. Salas gave a characterization of supercyclic bilateral backward weighted shifts via the Supercyclicity Criterion. This Criterion is an important tool in much recent works on supercyclic operators. Here, we want to extend a property of hypercyclic operators to a pair of commuting operators, and although the techniques work for any n-tuple of operators but for simplicity we prove our results only for the case $n=2$. For some topics we refer to [1-18].

\section{Main Results}

In this section, we prove that if a tuple $\mathcal{T}=\left(T_{1}, T_{2}, \ldots, T_{n}\right)$ is supercyclic and $\mathcal{F}_{\mathrm{T}}$ is a bounded set, then $T_{1}^{k(1)} T_{2}^{k(2)} \ldots T_{n}^{k(n)} \rightarrow 0$ as $k(i) \rightarrow \infty$ for $i=1, \ldots, n$.

Lemma 2.1. Let $\mathcal{T}=\left(T_{1}, T_{2}, \ldots, T_{n}\right)$ be a supercyclic tuple of continuous linear operators acting on a separable infinite dimensional Banach space $X$. If $S C(\mathcal{T}) \neq \varnothing$, then $S C(\mathcal{T})$ is dense in $X$.

Proof. Fix an enumeration $\left\{B_{n}: n \in \mathbb{N}\right\}$ of the open balls in $X$ with rational radii, and centers in a countable dense subset of $X$. By the continuity of the operators $T_{1}, T_{2}, \ldots, T_{n}$, the sets

$$
G_{j}=\bigcup\left\{\lambda T_{1}^{-k(1)} T_{2}^{-K(2)} \ldots T_{n}^{-K(n)}\left(B_{j}\right): k(i) \geq 0 ; i=1, \ldots, n ; \lambda \in \mathbf{C}\right\}
$$

are open. Since for any two non-void open sets $U$ and $V$, there exist $k(i) \geq 1$ for $i=1, \ldots, n$ and $\lambda \in \mathbf{C} \backslash\{0\}$ such that $\lambda T_{1}^{k(1)} T_{2}^{k(2)} \ldots T_{n}^{k(n)}(U) \cap V \neq \varnothing$, thus one can see that $S C(\mathcal{T})$ is exactly equal to $\bigcap\left\{G_{j}: j \in \mathbb{N}\right\}$ that is dense in $X$. This completes the proof.

Theorem 2.2. Let $\mathcal{T}=\left(T_{1}, T_{2}, \ldots, T_{n}\right)$ be supercyclic tuple of continuous linear operators acting on a separable infinite dimensional Banach space $X$. If $\mathcal{F}_{\mathbf{T}}$ is a bounded set, then $T_{1}^{k(1)} T_{2}^{k(2)} \ldots T_{n}^{k(n)} \rightarrow 0$ as $k(i) \rightarrow \infty$ for $i=1, \ldots, n$.

Proof. Since $\mathcal{F}_{\mathrm{T}}$ is a bounded set, there exists $M>0$, such that

$$
\left\|T_{1}^{k(1)} T_{2}^{k(2)} \ldots T_{n}^{k(n)}\right\| \leq M
$$


for all $k(i) \geq 0$ for $i=1, \ldots, n$. Let $x$ be a supercyclic vector for $\mathcal{T}$ such that

$$
\lim _{k(1), \ldots, k(n)} T_{1}^{k(1)} T_{2}^{k(2)} \ldots T_{n}^{k(n)} x \neq 0 .
$$

So there exists $r>0$ such that $\left\|T_{1}^{k(1)} T_{2}^{k(2)} \ldots T_{n}^{k(n)} x\right\|>r>0$ for every integers $k(i) \geq 0, i=1, \ldots, n$. Now, since $S C(\mathcal{T}) \neq \varnothing$, thus there exists a nonzero $y \in X$ with $\|y\|=1$ such that

$$
\lim _{k(1), \ldots, k(n)} T_{1}^{k(1)} T_{2}^{k(2)} \ldots T_{n}^{k(n)} y \neq 0 .
$$

Since $\operatorname{Orb}(\mathcal{T}, x)$ is dense in $X$, there exist sequences of scalars $\left\{c_{j}\right\} \subset \mathbb{C}$, $\left\{k_{j}(i)\right\}_{j} \subset \mathbb{N} ; i=1, \ldots, n$ such that $c_{j} T_{1}^{k_{j}(1)} T_{2}^{k_{j}(2)} \ldots T_{n}^{k_{j}(n)} x \rightarrow y$ as $j \rightarrow \infty$. Thus, there exists a natural number $k_{0}$ such that for all $j \geq k_{0}$ we have

$$
\left\|c_{j} T_{1}^{k_{j}(1)} T_{2}^{k_{j}(2)} \ldots T_{n}^{k_{j}(n)} x-y\right\|<1 / 2 .
$$

Thus $(2 M\|x\|)^{-1} \leq 2^{-1}\left\|T_{1}^{k_{j}(1)} T_{2}^{k_{j}(2)} \ldots T_{n}^{k_{j}(n)} x\right\|^{-1}<\left|c_{j}\right|$ for all $j \geq k_{0}$. Define $\alpha=r\left(3 M^{2}\|x\|\right)^{-1}$. Since $y$ is a supercyclic vector for $\mathcal{T}$, there exists $j_{0}>k_{0}$, $c_{j_{0}} \in \mathbb{C}$, and $k_{j_{0}}(i) \in \mathbb{N} ; i=1, \ldots, n$ such that

$$
\left\|c_{j_{0}} T_{1}^{k_{j_{0}}(1)} T_{2}^{k_{j_{0}}(2)} \ldots T_{n}^{k_{j_{0}}(n)} x-y\right\|<\alpha / 2 .
$$

Since

$$
\lim _{k(1), \ldots, k(n)} T_{1}^{k(1)} T_{2}^{k(2)} \ldots T_{n}^{k(n)} y \neq 0,
$$

thus for some $k(1), k(2), \ldots, k(n) \in \mathbb{N}$, we have $\left\|T_{1}^{k(1)} T_{2}^{k(2)} \ldots T_{n}^{k(n)} y\right\| \leq M \alpha / 2$. Put

$$
L=c_{j_{0}} T_{1}^{k_{j_{0}}(1)+k(1)} T_{2}^{k_{j_{0}}(2)+k(2)} \ldots T_{n}^{k_{j_{0}}(n)+k(n)} x-T_{1}^{k(1)} T_{2}^{k(2)} \ldots T_{n}^{k(n)} y .
$$

So we get

$$
\begin{aligned}
\|L\| & =\left\|T_{1}^{k(1)} T_{2}^{k(2)} \ldots T_{n}^{k(n)}\left(c_{j_{0}} T_{1}^{k_{j_{0}}(1)} T_{2}^{k_{j_{0}}(2)} \ldots T_{n}^{k_{j_{0}}(n)} x-y\right)\right\| \\
& \leq\left\|T_{1}^{k(1)} T_{2}^{k(2)} \ldots T_{n}^{k(n)}\right\|\left\|c_{j_{0}} T_{1}^{k_{j_{0}}(1)} T_{2}^{k_{j_{0}}(2)} \ldots T_{n}^{k_{j_{0}}(n)} x-y\right\| \\
& <M \alpha / 2 .
\end{aligned}
$$

Note that

$$
\begin{aligned}
r(2 M\|x\|)^{-1} & \leq\left\|c_{j_{0}} T_{1}^{k_{j_{0}}(1)} T_{2}^{k_{j_{0}}(2)} \ldots T_{n}^{k_{j_{0}}(n)} x\right\| \\
& \leq\left\|c_{j_{0}} T_{1}^{k_{j_{0}}(1)} T_{2}^{k_{j_{0}}(2)} \ldots T_{n}^{k_{j_{0}}(n)} x-T_{1}^{k(1)} T_{2}^{k(2)} \ldots T_{n}^{k(n)} y\right\|
\end{aligned}
$$




$$
\begin{aligned}
& +\left\|T_{1}^{k(1)} T_{2}^{k(2)} \ldots T_{n}^{k(n)} y\right\| \\
& \leq r(3 M\|x\|)^{-1}
\end{aligned}
$$

that is a contradiction. Thus, indeed $T_{1}^{k(1)} T_{2}^{k(2)} \ldots T_{n}^{k(n)} \rightarrow 0$ as $k(i) \rightarrow \infty$ for $i=1, \ldots, n$ whenever $x \in S C(\mathcal{T})$. Now, since $S C(\mathcal{T})$ is dense in $X$, thus it should be $T_{1}^{k(1)} T_{2}^{k(2)} \ldots T_{n}^{k(n)} \rightarrow 0$ as $k(i) \rightarrow \infty$ for $i=1, \ldots, n$ for all $x \in X$. This completes the proof.

\section{References}

[1] S.I. Ansari, P.S. Bourdon, Some properties of cyclic operators, Acta Sci. Math., 63 (1997), 195-207.

[2] F. Bayart, E. Matheron, Dynamics of Linear Operators, Cambridge University Press (2009).

[3] J. Bes, A. Peris, Hereditarily hypercyclic operators, J. Func. Anal., 167, No. 1 (1999), 94-112.

[4] N.S. Feldman, Countably hypercyclic operators, Journal of Operator Theory, 273 (2002), 67-74.

[5] N.S. Feldman, Hypercyclic tuples of operators and somewhere dense orbits, J. Math. Appl., 346 (2008), 82-98.

[6] H.M. Hilden, L.J. Wallen, Some cyclic and non-cyclic vectors of certain operators, Indiana Univ. Math. J., 24 (1974), 557-565.

[7] H.N. Salas, Supercyclic Subspace: Spectral theory and weighted shifts, Advances in Mathematics, 163 (2001), 74-134.

[8] H.N. Salas, Supercyclicity and weighted shift, Studia Mathematica, 135 (1999), 55-74.

[9] B. Yousefi, H. Rezaei, Hypercyclicity on the algebra of Hilbert-Schmidt operators, Results in Mathematics, 46 (2004), 174-180.

[10] B. Yousefi, H. Rezaei, Some necessary and sufficient conditions for Hypercyclicity Criterion, Proc. Indian Acad. Sci. (Math. Sci.), 115, No. 2 (2005), 209-216. 
[11] B. Yousefi, A. Farrokhinia, On the hereditarily hypercyclic vectors, Journal of the Korean Mathematical Society, 43, No. 6 (2006), 1219-1229.

[12] B. Yousefi, H. Rezaei, J. Doroodgar, Supercyclicity in the operator algebra using Hilbert-Schmidt operators, Rend. Circ. Mat. Palermo, Tomo LVI, No. 2 (2007), 33-42.

[13] B. Yousefi, H. Rezaei, Hypercyclic property of weighted composition operators, Proc. Amer. Math. Soc., 135, No. 10 (2007), 3263-3271.

[14] B. Yousefi, S. Haghkhah, Hypercyclicity of special operators on Hilbert function spaces, Czechoslovak Mathematical Journal, 57, No. 132 (2007), 1035-1041.

[15] B. Yousefi, H. Rezaei, On the supercyclicity and hypercyclicity of the operator algebra, Acta Mathematica Sinica, 24, No. 7 (2008), 1221-1232.

[16] B. Yousefi, R. Soltani, Hypercyclicity of the adjoint of weighted composition operators, Proc. Indian Acad. Sci. (Math. Sci.), 119, No. 3 (2009), 513-519.

[17] B. Yousefi, J. Izadi, Weighted composition operators and supercyclicity criterion, International Journal of Mathematics and Mathematical Sciences, 2011, doi: $10.1155 / 2011 / 514370$ (2011).

[18] B. Yousefi, Hereditarily transitive tuples, Rend. Circ. Mat. Palermo, 2011, doi: 10.1007/s12215-011-0066-y (2011). 
